\title{
La prospection géophysique des eaux souterraines
}

\section{Geophysical ground water prospection}

\author{
PAR J.-J. BREUSSE, \\ DIRECTEUR A LA COMPAGNIE GÉNÉRALE DE GÉOPHYSIQUE, \\ PARIS
}

\begin{abstract}
Court historique des applications de la géophysique d̀ la recherche de l'eau.

Méthodes mises en cuvre : prospection électrique avec ses techniques du sondage électrique et de la carte de résistivité; sismique réfraction. Principaux problèmes traités en hydrologie. Exemples présentés :

- Catane (Italie): étude d'une plaine alluviale par sondages électriques;

- Sbeitla (Tunisie) : étude d'un seuil hydraulique par résistivité;

- Warnier (Algérie) : étude par sondages électriques de l'allure d'une couche aquifère profonde;

- Elavagnon (Togo): recherche d'eau dans un substratum cristallophyllien par carte de résistivite;

- Bassari (Togo): recherche d'eau dans des schistes par carte de résistivité;

- Benguerir (Maroc) : recherche d'eau par sondages électriques pour une base américaine.
\end{abstract}

La recherche des eaux souterraines, parallèlement à celle des minerais et hydrocarbures, a donné lieu à des études qui ont abouti à la mise au point de certaines méthodes géophysiques dont l'utilisation est actuellement bien répandue. Ces méthodes et quelques exemples d'application font l'objet de ce mémoire.

Historiquement, c'est l'invention et la mise au point par C. et M. Schlumberger de la prospection électrique qui a conduit aux premières études hydrologiques. On sait que la recherche de l'eau, même dans le cas le plus fréquent et le plus simple des nappes alluviales, est liée à

\begin{abstract}
This article includes a brief historical review of the application of geoplysical methods to water prospection, a description of methods used-i.e. electrical prospection by means of electrical soundings and resistivily charts, and the seismic refraction method. The main problems dealt with in hydrology are discussed, with the following examples :

- Catania, Italy : A survey of an alluvial plain by electrical soundings.

- Sbeitla, Tunisie : A study of a sill by the resistivity method.

- Warnier, Algeria : A survey of a deep aquifer by electrical soundings.

- Elavagnon, Togo : Prospection for water in a crystallophyllian substratum by the resistivity chart method.

- Bassari, rogo: Prospection for water in shale by the resistivity chart method.

- Benguerir, Morocco : Prospection for water for an American base by electrical soundings.
\end{abstract}

l'allure des couches, à leur faciès et à une question de perméabilité. Le sondage électrique, qui permet de suivre les formations, de déterminer leur épaisseur et jusqu'à un certain point leur faciès ainsi que les zones les plus perméables, a été l'outil décisif et remarquable pour cette application.

\section{Méthodes mises en œuvre :}

Présentons les principales méthodes de prospection : électrique, sismique réfraction.

En prospection électrique, on sait que la résis- 
tivité d'un terrain dépend beaucoup des propriétés physiques et chimiques de l'eau d'imbibition et de sa teneur, d'où les larges variations de ce «paramètre $»$, de 0,1 à plusieur's milliers d'ohms. Elle permet de comparer, qualitativement tout au moins, les perméabilités des alluvions et formations meubles. C'est ainsi que, pour une même eau d'imbibition, une alluvion sera d'autant plus perméable qu'elle sera électriquement résistante, tandis que, dans une masse compacte, une zone faillée se marquera par une bande conductrice.

Le sondage électrique mesure la résistivité apparente en fonction de la profondeur. La carte de résistivité, au contraire, la fait connaître, à profondeur constante, sur tout un périmètre. La prospection électrique se distingue par une grande facilité de mise en œuvre. Elle est très souple, s'adapte immédiatement à des problèmes variés el conduit à des économies impor- tantes par la suppression des forages mal placés (économie de 50 à $70 \%$ selon des chiffres présentés à la Conférence Inter-Africaine d'Hydrologie, Nairobi, janvier 1961). Elle s'appuie sur une interprétation mathématique, donc scientifique, des diagrammes.

On a songé à utiliser les courants naturels de la polarisation spontanée, causés par les phénomènes d'électrofiltration et d'évaporation à la surface des terrains détritiques et de certains massifs de roches poreuses. En reconnaissance générale, la polarisation spontanée aiderait à localiser des zones de plus grand coefficient d'infiltration de l'impluvium et même à repérer des nappes peu profondes. L'expérimentation mériterait d'être poussée en pays tropicaux et semidésertiques. En ce qui concerne la polarisation provoquée, les essais pratiques n'ont pas encore été très probants, les phénomènes étant surtout fonction de la teneur en argile des alluvions.
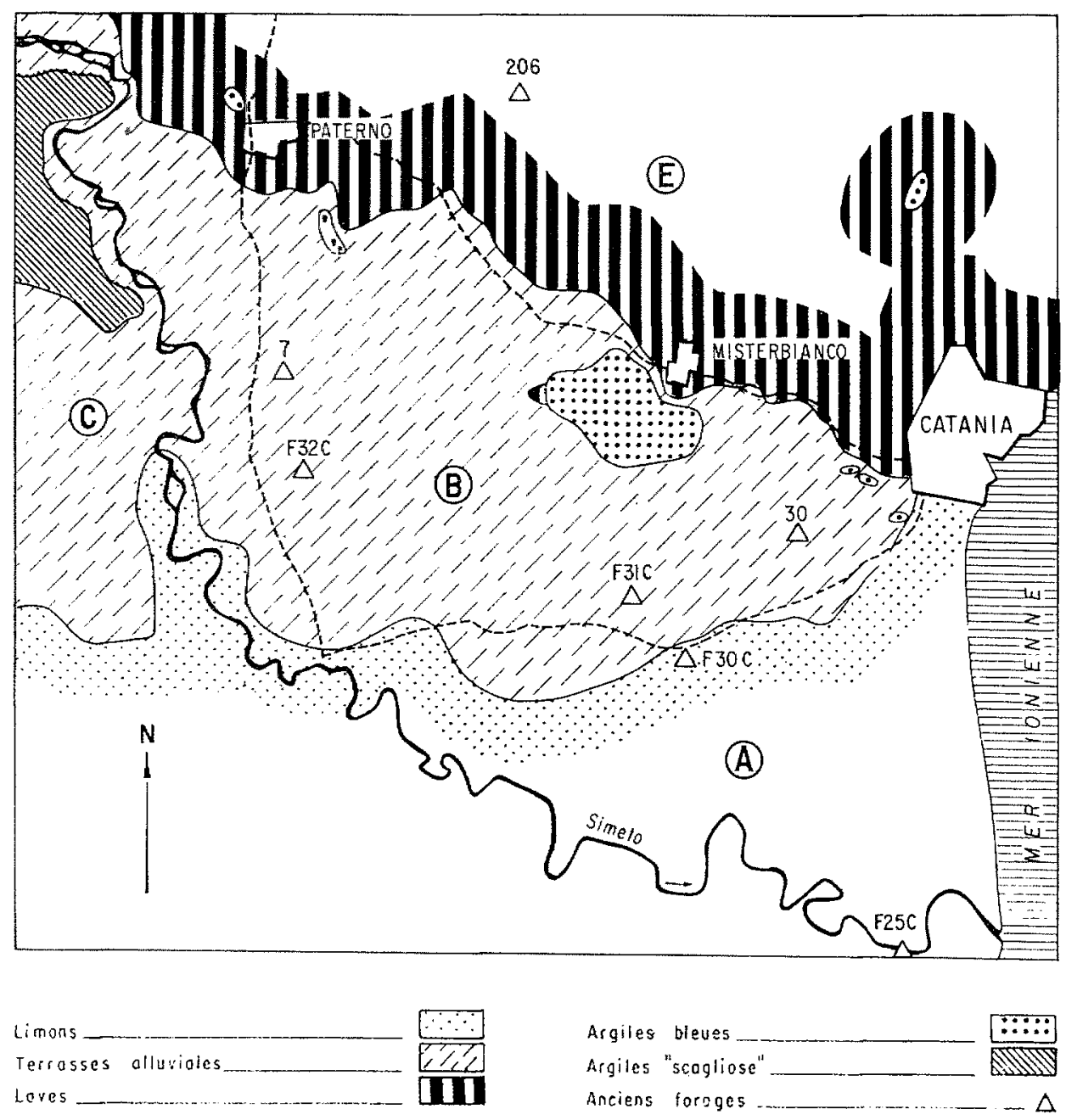

Fig. 1

PIaine de Catane Carte géologique. 
La sismique réfraction met en jeu les vitesses de propagation des ondes sismiques. On l'emploie surtout à reconnaître, sous des alluvions, l'allure du rocher; elle vient au secours de la méthode électrique qui est en difficulté lorsque les résistivités des alluvions et du rocher sont trop voisines. Pour des études structurales profondes (plusieurs centaines de mètres) la sismique réfraction intervient dans des cas particuliers, par exemple étude d'un horizon calcaire aquifère. Comparée à la prospection électrique, la sismique réfraction est beaucoup moins sensible aux faciès des terrains, à leur altération et ne fournit pas d'indications de la perméabilité. Sa mise en wuvre est plus complexe, son prix de revient est plus élevé. Elle constitue done une méthode d'appoint en général.

Pour mémoire, citons la méthode magnétique, efficace sur les formations volcaniques, pour reconnaître, par exemple, des coulées de lave ou des dykes.

Examinons maintenant les différents problèmes qui sont posés couramment au géophysicien. En premier lieu, viennent les études des nappes alluviales et des dunes. Il s'agit d'estimer des épaisseurs d'alluvions, de découvrir des anciens lits et sur leur tracé, localiser les zones les plus perméables, de connaître le substratum, de discerner les formations imbibées d'eau, d'en apprécier la salinité, de localiser les limites entre nappes d'eaux douces ou salées.

Des problèmes techniques sont également posés : préciser en profondeur l'allure d'un terrain qui, connu en affleurement, se révèlera aquifère et peut-être artésien. On étudie les failles, horst et grabens, anticlinaux et synclinaux et surtout les seuils hydrauliques. En présence de formations volcaniques on met en évidence les coulées de laves et les tufs perméables avec l'ancien réseau hydrographique.

En résumé, peu de problèmes échappent à l'investigation géophysique, hormis ceux que soulève le véritable registre karstique des eaux dans les calcaires. Mention doit être faite ici du carottage électrique. Il est devenu une aide indispensable à la prospection des formations hydrauliques par sondage et son exécution accompagne de plus en plus systématiquement les forages de prospection.

\section{Citons quelques exemples de prospection hydrologique :}

Plaine de Catane (ITalie). - Cet exemple se rapporte à l'étude hydrologique générale de la plaine de Catane dont la superficie dépasse $300 \mathrm{~km}^{2}$ : une partie est couverte par des coulées de basalte (fig. 1), le reste du bassin par des alluvions : alluvions grossières et en terrasses, masquées, ou non, par des limons argileux. Ces formations reposent sur des argiles bleues quaternaires et tertiaires constituant le plancher imperméable, au-dessus du tertiaire sous-jacent déjà connu sur les bordures.

De nombreuses explorations s'étant révélées stériles (forages $7,30 c, 31 c$ et $32 c$ ), il a été demandé à la méthode électrique d'éclaicir les possibilités aquifères du tertiaire sous-jacent et de certains bancs sableux intercalés dans les argiles bleues quaternaires ct de préciser le tracé de l'ancien réseau hydrographique. Quelques sondages électriques ont montré qu'il existait sous le recouvrement alluvial volcanique, une formation conductrice correspondant à des épaisseurs d'argiles bleues, beaucoup plus importante qu'on ne l'imaginait. Ils ont également indiqué que les banes sableux étaient trop minces ou trop imbibés d'eau salée. II restait à préciser le tracé de l'ancien réseau hydrographique. Grâce à de nouveaux sondages électriques, il a été construit une carte en courbes de niveau du toit des argiles bleues (fig. 2). Cette carte montre que le massif argileux culmine au nord de l'étude. Il est entaillé par un puissant réseau hydrographique qui, sous les laves, collecte la majorité des eaux.

Les forages exécutés à la suite de ces conclusions ont été couronnés de succès. Ceux qui ont été implantés sur le lit de l'ancien fleuve Simeto, ont rencontré de puissantes nappes artésiennes.

Actuellement le débit total d'une vingtaine de forages dont nous connaissons les positions atteint $300 \mathrm{I} / \mathrm{s}$.

Seurl hydraulique de Sbertha (Tunisie). Du Miocène gréseux à intercalations marneuses repose sur une série marno-calcaire du Crétacé supérieur, dont les bancs calcaires se comportent probablement comme des collecteurs d'une partie des nappes miocènes. Les relevés géologiques laissaient prévoir la présence d'une grande faille capable de constituer un seuil hydraulique.

L'étude géophysique a commencé par l'exécution d'un petit nombre de S.E. en $A B=2000 \mathrm{~m}$, dont les S.E. 1 et 2 (fig. 3) sont très suggestifs, On constate en effet la présence au. S.E. 1 d'un horizon conducteur épais qui. est absent au S.E. 2. En conséquence, ces S.E. mettent bien en évidence deux compartiments séparés par une faille. Le compartiment est est caractérisé par une forte épaisseur de Miocène gréseux dont la résistîvité est de $150-200 \mathrm{ohm}$. m, alors que dans le compartiment ouest apparaissent les marnes et marno-calcaires du Crétacé sous une centaine de mètres de Miocène.

L’étude du tracé de la faille a été réalisée à l'aide de onze profils de résistivité en ligne $A B$ de $1000 \mathrm{~m}$. La carte de résistivité correspondante fait l'objet de la figure 4 sur laquelle les 


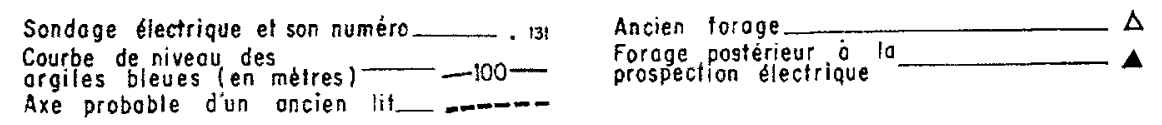

Axe probable d'un ancien lit

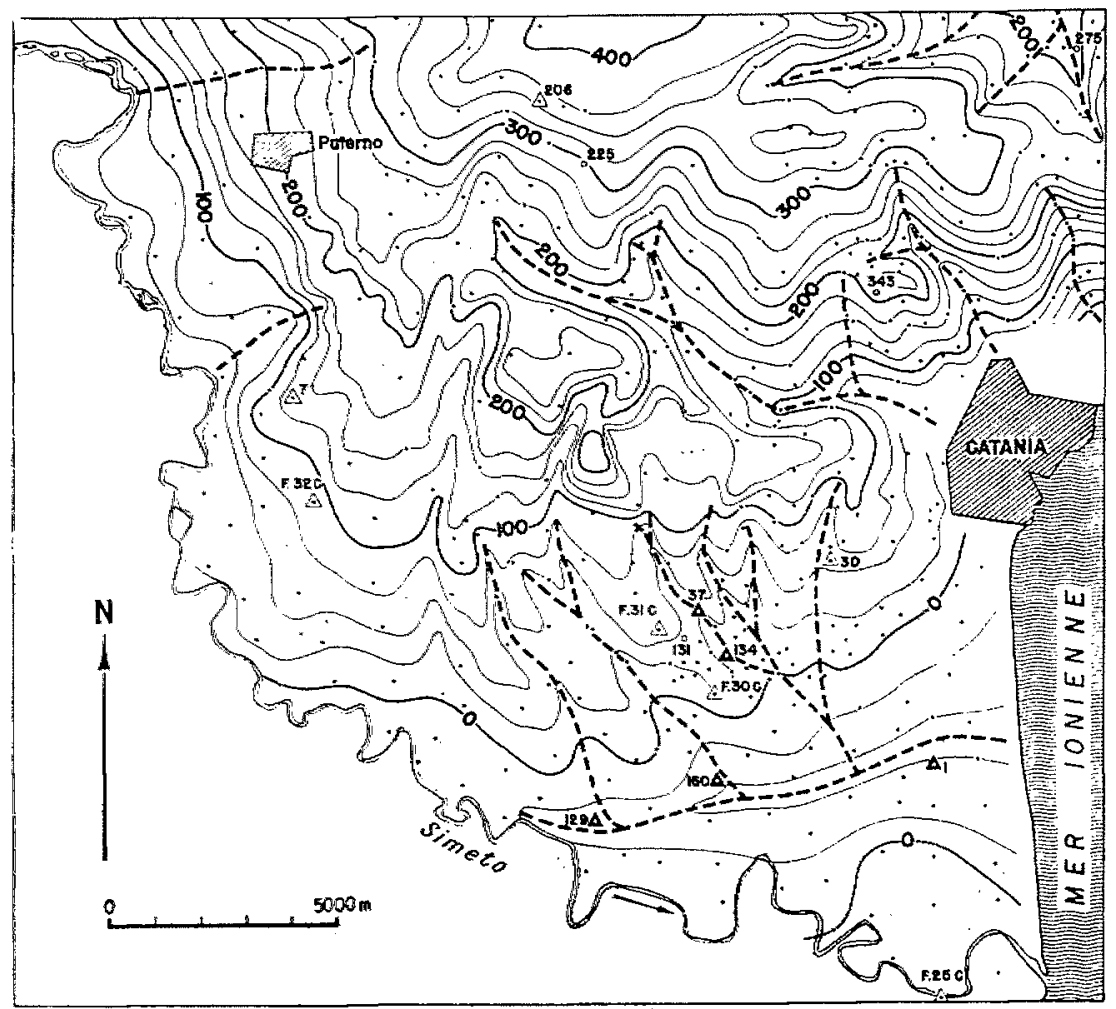

EXEMPLES DE SONDAGES ELECTRIQUES
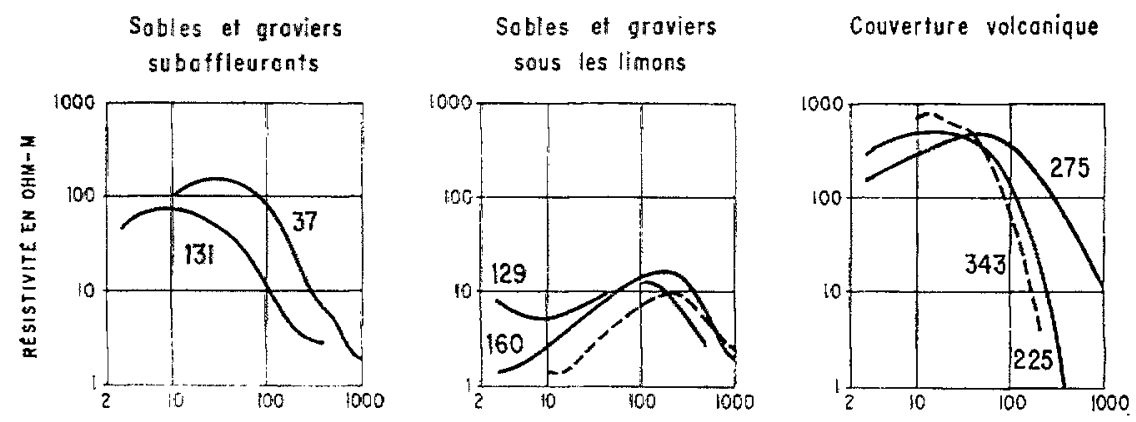

Fig. 2

Plaine de Catane. Carte du toit $A B$ EN m des argiles bleues (substratum). courbes d'équirésistivité ont été tracées de 50 en $50 \mathrm{ohm} . \mathrm{m}$. Les deux compartiments sont bien caractérisés : conducteur à l'ouest et résistant à l'est et la brusque remontée de la résistivité sur les profils permet de tracer la faille F 1 du seuil hydraulique avec précision. Un second accident transversal moins important $F 2$ a été mis en évidence.

Une seconde carte de résistivité (fig. 5) a été établie à partir des mêmes profils, mais exécutés avec une ligne $A B$ de $400 \mathrm{~m}$ afin d'étudier le comportement du Miocène dans le compartiment ouest. Cette carte montre l'apparition de la partie nord d'une lentille particulièrement résis- tante, donc gréseuse et assez peu épaisse, car elle n'est plus qu'à peine visible sur la carte de résistivité en $\mathrm{AB}=1000 \mathrm{~m}$. On a pensé que cette lentille pouvait, outre les bancs calcaires, présenter un intérêt aquifère et un forage a été implanté au S.E. 1. Il a bien rencontré $120 \mathrm{~m}$ de grès aquifères avec eau jaillissante; il a été ensuite poussé jusqu'aux calcaires qui ont été tonchés à $360 \mathrm{~m}$ de profondeur avec un excellent débit et un artésianisme de plus de $10 \mathrm{~m}$.

BaSE aMÉRICAINE DE LA PLAINE DE BENGUERIR (MAROC). - La localité de Benguérir est située à $70 \mathrm{~km}$ au nord de Marrakech sur la route de Marrakech à Casablanca. Au sud de Benguerir, 


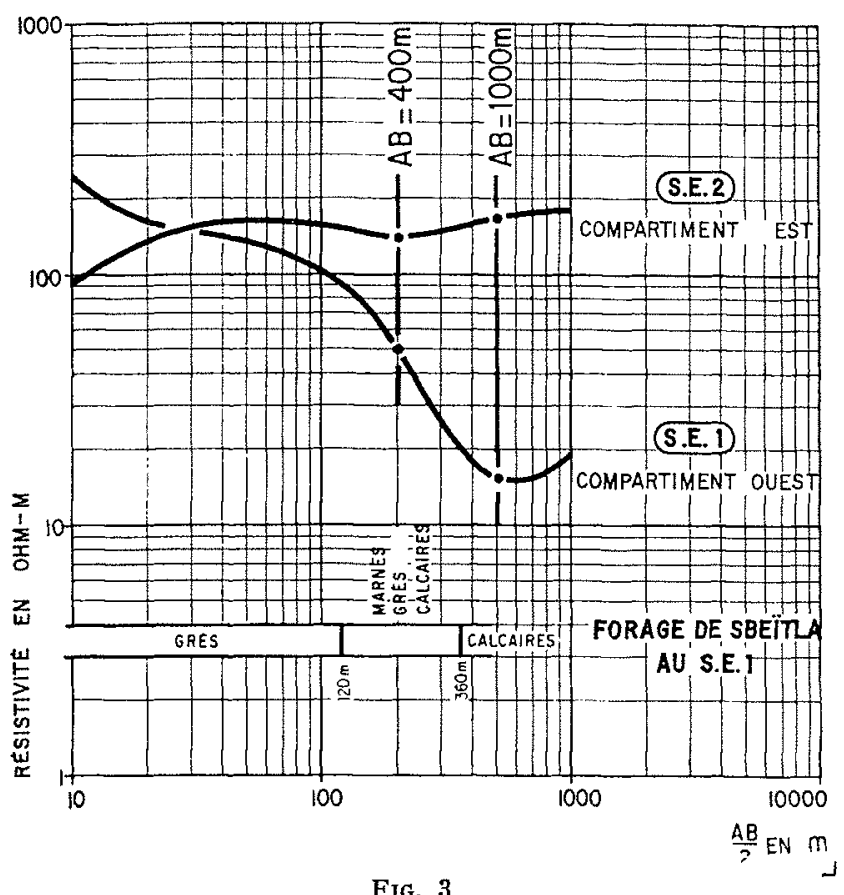

Sbeitla.

Exemples de sondages électriques.

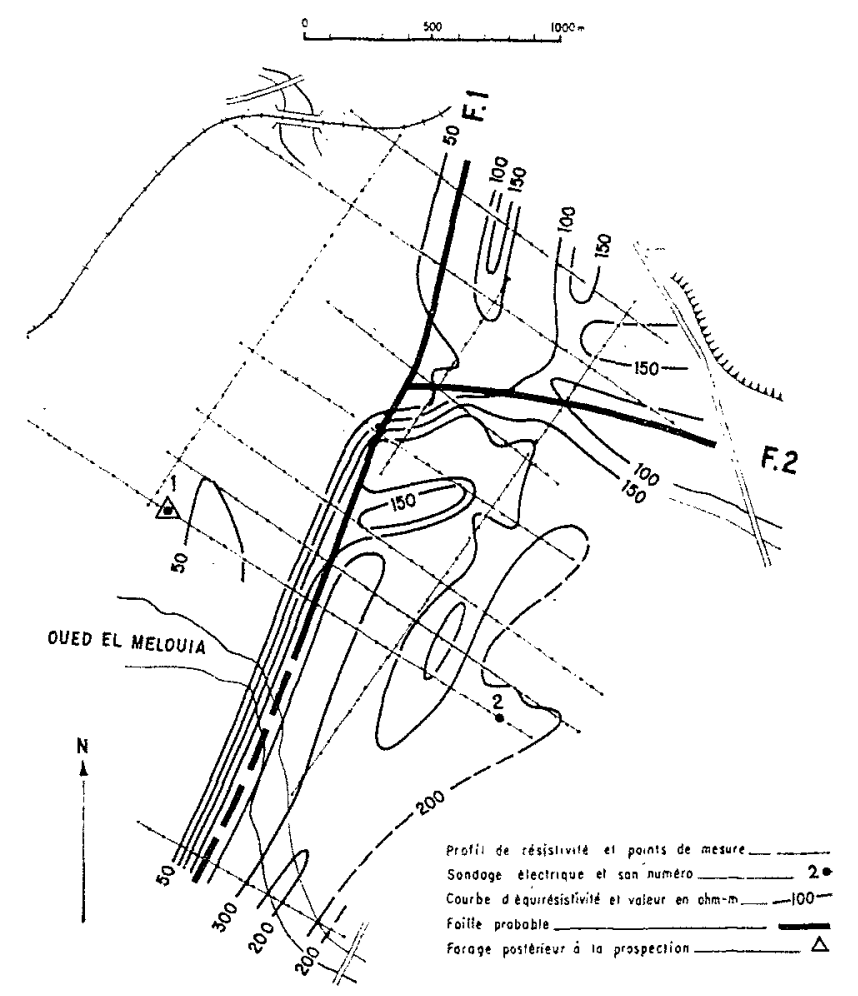

FIG. 5

Sbeitla.

Carte de résistivité $(A B=400 \mathrm{~m})$.

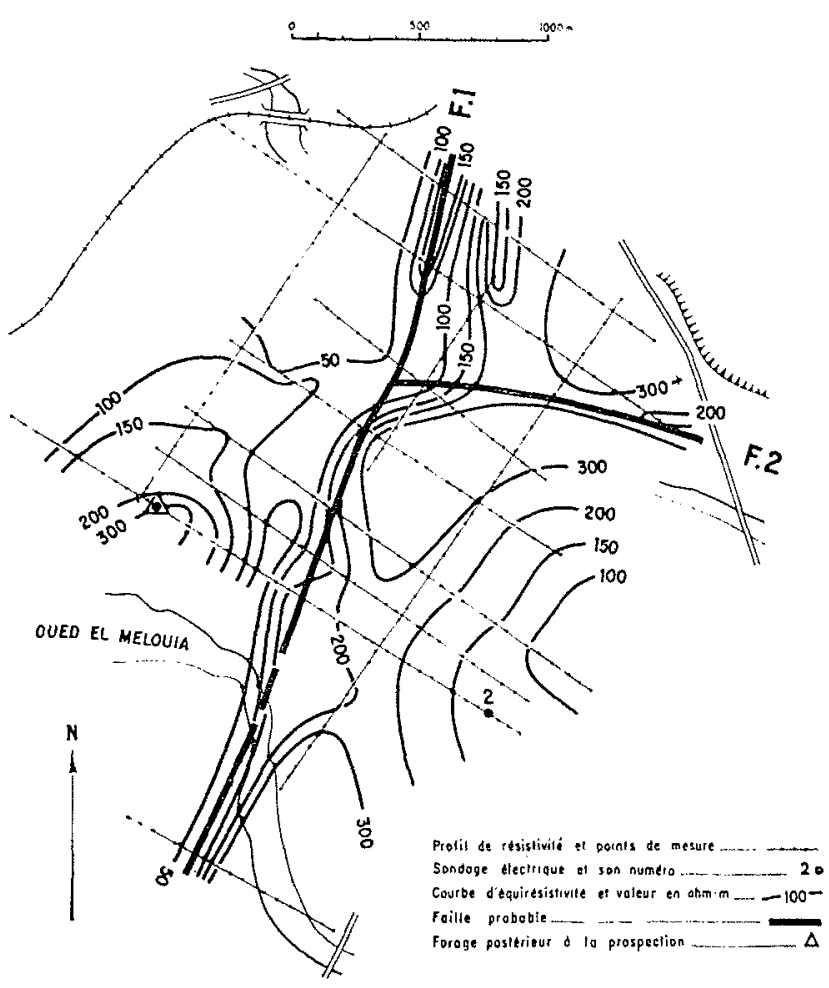

FIG. 4

Sbeitla.

Carte de résistivité $(A B=1000 \mathrm{~m})$.

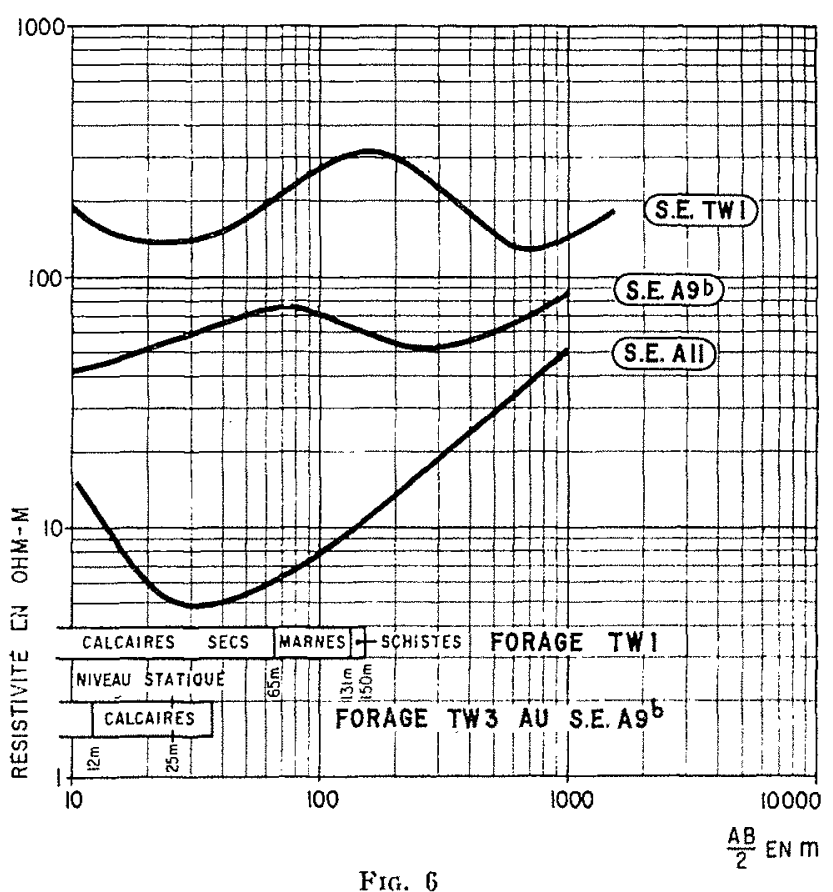

Fxg. 6

Benguerit.

Exemples de sondages électriques. 


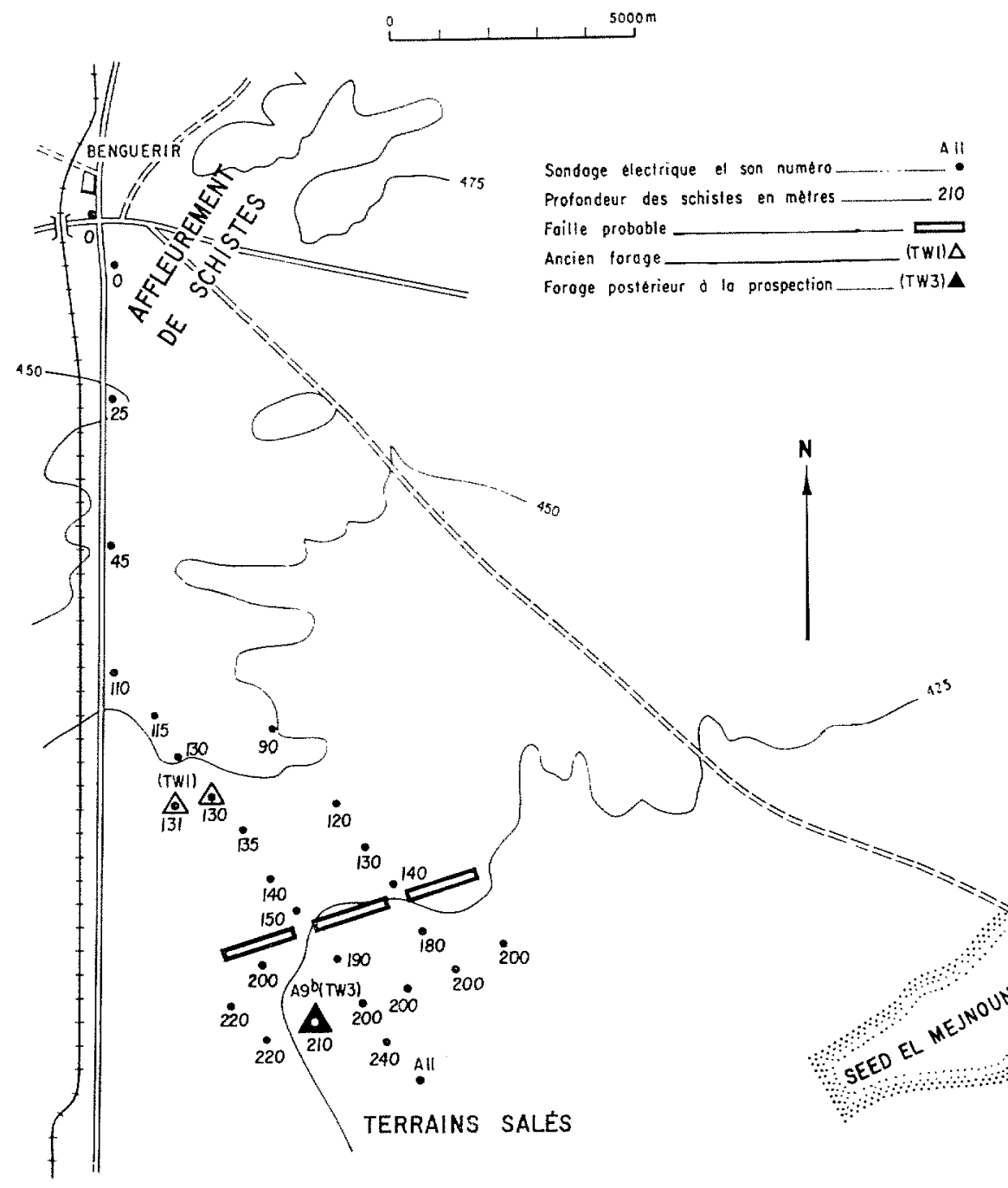

Fig. 7

Benguerir.

Carte des résultats.

s'étend une plaine à peu près inculte et dont une partie avait été choisie pour la construction d'une base aérienne américaine.

$\mathrm{Au}$ point de vue géologique, le socle de la plaine est constitué par des schistes primaires avec un recouvrement de marnes et calcaires crétacés et des calcaires néogènes surmontés par des limons quaternaires. Pour l'alimentation en eau de la base, deux forages, dont TW 1 arrèté dans les schistes à $150 \mathrm{~m}$ de profondeur, étaient entièrement négatifs.

La question de la recherche d'eau pour la future base se posait d'urgence. II se trouvait qu'une équipe de prospection électrique travaillait au Maroc en permanence et il lui fut demandé d'étudier en priorité ce problème. Deux profils de $40 \mathrm{~km}$ de long, distants entre eux de $2 \mathrm{~km}$ et comprenant des sondages électriques, tous les kilomètres environ, furent exécutés en s'appuyant au nord sur les effleurements de schistes de Benguerir.

Le S.E. en $A B=3000 \mathrm{~m}$ effectué sur le forage
TW 1 a montré que la résistivité des calcaires secs est voisine de $400 \mathrm{ohm} . \mathrm{m}$ (fig. 6). L'horizon conducteur sous-jacent correspond aux marnes et la remontée finale aux schistes du socle. Il a été ainsi possible d'estimer la profondeur des schistes et de déterminer leur tectonique (fig. 7). Les schistes s'ennoient vers le sud et une faille $\mathrm{F} 1$ mise en évidence sur les deux profils et d'une cinquantaine de mètres de rejet les effondre au sud.

Si l'on examine maintenant le S.E. A9 $b$ appartenant au compartiment sud (fig. 6) on constate qu'il indique la présence d'un horizon résistant surmontant les marnes. La conclusion a été qu'il s'agissait bien des calcaires peu profonds, mais probablement aquifères, vu leur résistivité relativement faible. Le forage TW 3 implanté sur le S.E. A9b a rencontré les calcaires à $12 \mathrm{~m}$ de profondeur avec un débit de $10 \mathrm{~m}^{3} / \mathrm{h}$. Cette nappe importante et d'assez bonne qualité a été exploitée par quatre autres forages situés dans la même zone, ce qui résolvait le problème. Il est 
certain que la salinité de l'eau $(1 \mathrm{~g} / 1$ de $\mathrm{NaCl})$ explique la baisse de résistivité importante des calcaires lorsqu'ils sont aquifères.

Vers le sud, la prospection électrique a montré (fig. 6) (S.E. A11) que l'on était en présence d'un recouvrement dans lequel la salure des terrains augmente et s'étend jusqu'à une profondeur importante et qu'en conséquence cette région était sans intérêt. La durée d'exécution de l'étude a été de deux semaines.

De nombreux exemples illustrant les différents problèmes hydrologiques énoncés au début de cet article pourraient être également présentés (séparation des eaux douces et saumâtres, recherches dans un système volcanique ou dans un substratum éruptif ou cristallophyllien, etc.), mais nous avons dû y renoncer faute de place.

\section{En conclusion :}

Ainsi que pour les études pétrolières où l'intervention de la géophysique n'est plus discutée depuis longtemps, on constate que l'on a de plus en plus recours à elle pour les recherches hydrologiques. Certes, on ne saurait cependant pousser trop loin la comparaison, car si les études pétrolières permettent l'utilisation de méthodes géophysiques coûteuses, il n'en est pas de même pour l'eau, dont les investissements mis en jeu et leur rentabilité sont loin d'être à la même échelle. Aussi convient-il d'adopter des méthodes géophysiques légères, efficaces, d'une application aussi générale que possible et avant tout à bon marché et je pense, en disant cela, en tout premier lieu à la prospection électrique qui réunit tous ces caractères.

\section{DIS CUSSION}

Président : M. BECKER

M. le Président remercie M. Breusse qui a donné un aperçu précis et illustré de nombreux exemples de l'application de la géophysique à la déconverte des nappes souterraines.

En ce moment où la comparaison entre les besoins et les ressources donne certaines inquiétudes, M. le Président insiste sur le très grand intérêt de la prospection géophysique des eaux souterraines, comme moyen d'investigation de nouvelles sources d'eau.

Il semble à M. Mauchamp que la prospection électrique pour la recherche des nappes souternaines soit identique à celle pour la connaissance géologique en vue de la détermination $\mathrm{du}$ toit du substratum sous-alluvial. Dans ce cas, une reconmaissance géologique peutelle ètre soumise à une nouvelle interprétation pour déterminer la carte de la perméabilité des alluvions? Est-ce uniquement une question d'interprétation complémentaire?

M. Breusse indique que la perméabilité se détermine uniquement par la mesure de la résistivité. On peut mesurer par le caleul la valeur du $\mathrm{H}_{\mathrm{Q}}\left(^{*}\right)$; du point de rue hydrologique, ce sont les alluvions dont le produit $H_{Q}$ sera le plus élevé qui seront les meilleures (grande épaisseur on grande résistivité).

On obtient donc une valeur, non absolue, mais seulement relative, de la perméabilité des diverses zones

(*) Où $H$ est l'épaisseur des alluvions et $\rho$ leur résistivité. perméables, mais cela suttira pour connaitre la meilleurc zone où peut être fait le captage.

M. MaUchamp demande si avec des essais de perméabilité par pompage on ne pourrait pas étalonner les valeurs relatives données dans les conditions préctdentes par la Géophysique.

M. Breusse indique :

1" que chaque terrain a une résistivite propre;

2 "qu'il n'a jamais fait des essais de perméabilite par pompage pour l'interprétation des perméabilités et l'étalonnage de la prospection;

$3^{\circ}$ qu'il vaudrait mieux travailler la relation entre la résistivité ou le produit $H_{p}$ et la perméabilité en faisant des essais de pompage, mais jusqu'à présent les clients ne communiquement malheureusement pas les essais de pompage.

M. Bourrier demande si les méthodes de prospection électrique sont adaptables à la mesure de l'humidité du sol, pour la conduite des irrigations.

M. Breusse indique que, dans ce cas, on prend unc ligne d'émission de courant $A B$ de 5 ou $10 \mathrm{~m}$. Aves cette méthode, on est maître de la profondeur d'investigation. En gros, la profondeur atteinte est de l'ordre du quart de la ligne d'émission $A B$, et il est possible de dresser des cartes de résistivité correspondant à différentes profondeurs, ce qui donnera une idée du degré d'humidité du sol.

M. le Président remercie à nouveau $M$. Breusse. 